\title{
Metastability in the brain
}

\author{
J.A.S. Kelso, E. Tognoli
}

\begin{abstract}
Metastability is a property appearing in a system of non-linearly coupled non-linear oscillators wherein the units exhibit simultaneously a tendency to function autonomously (local segregation) and a tendency for coordinated activity (global integration). This property has been observed to emerge in systems with broken symmetry (different intrinsic frequencies of the constituent oscillators) when a threshold of the balance between local and global coupling strength is reached. Such a system provides an optimal flexibility, ie. the system presents attraction without attractor and can rapidly shift from one tendency to another. We hypothesize here that metastability is a principle underlying neuro-cognitive organization.
\end{abstract}

\section{INTRODUCTION}

I $\mathrm{T}$ is now widely accepted that the brain is a self-organized system. In general, systems formed by multiple quasi-identical units can adopt various states. Among others, simple examples are relaxation to uniform level of activity (a state with no spatial variability and with a monotonic temporal dynamic), or gradients (a state with no temporal variability but with the organization of a monotonic change in space).

"Patterns" are transient forms of organization of a system presenting repeating structures in the

This work was supported in the US Office of Naval Research. J.A.S. Kelso is the Glenwood and Martha Creech chair of Science, and professor in the Human Brain and Behavior Laboratory at Center For Complex Systems and Brain Sciences, Florida Atlantic University, 777 Glades Road FL 33431 USA (561-297-2230; fax: 561-297-3634; e-mail: kelso@ccs.fau.edu).

E. Tognoli is a post doctoral fellow in the Human Brain and Behavior Laboratory at Center For Complex Systems and Brain Sciences, Florida Atlantic University, 777 Glades Road FL 33431 USA (561-297-0110; fax: 561-297-3634; e-mail: tognoli@ccs.fau.edu). time and space domains. They are taking place in a sparsely and anisotropically connected network of neurons.

Further postulates on the dynamics of succession between patterns led us to a theory of metastability in (large-scale) neurocognitive networks [1], [2]. This view was later developed in several other research groups [3], [4], [5].

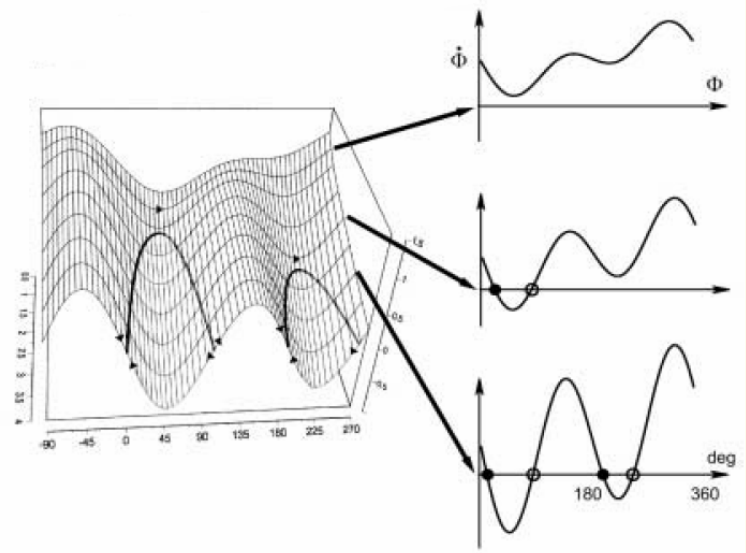

Fig. 1. a behavioral model of coordination. For increasing values of a parameter, point attractors (bottom right) are successively annihilated (middle right) until eventually only remnants of the past attractors are present (top right)

The property of metastable systems has first been investigated in behavioral systems [6], [7]. Using theoretical modeling of those systems, it was shown that metastable states emerged for a critical threshold of the balance between intrinsic and extrinsic coupling strength (see figure 1). In a simulated neuronal system, Friston [3] demonstrated that measures of metastability presented a non-monotonical $U$ curve, reaching a maximum when the extrinsic connectivity is 
present but sparse, and disappearing for global coherence when the extrinsic connectivity reaches higher values. Recent quantitative estimations of the cortical connectivity reinforced this hypothesis: mathematical estimation are incompatible with a dense cortical connectivity matrix at the macroscale [8], [9].

\section{CRITICALITY}

Similar to Friston's simulation study [3], the bounded pervasiveness of large-scale brain coherence is observed in the biological brain.

In the complex cognitive architecture, both cues of organization and fluctuations are observable. These two poles situate the brain system as intermediary between completely determined architectures where adaptation to infinite range of environmental changes is not possible and completely dissipative architectures where stability is not possible [10]. These boundaries have been more recently conceptualized in term of criticality [11] (see figure 2).

Stability is enrooted in the philogenetic and ontogenetic constraints exerted over the structural and functional connectivity of the brain [12]. Only a subset of all the possible configurational states of $10^{14}$ neurons constituting the human brain can be expressed, owing to the mutual constraints existing at the local and global scales. The tradeoff of stability, quantitatively, is to allow enough activation so as to enable the selective propagation of information at a large scale relevant for cognition, without invading the totality of the network, where any computational potential would be lost. Loss of computational potential is seen in some pathological states such as generalized epilepsy, where the totality of the network is transiently kindled into hypersynchronous firing, and where any sign of cognitive activity including consciousness is lost [13].

On the other hand, fluctuations or "noise" are a necessary feature of brain organization [10], [1], [14]. A stable system, at best, would stay entrapped to some stereotyped successions of reactive states and would be rendered unable to produce any kind of new or adaptative behavior [15]. At worse, it would reach equilibrium. We suggest that the balance between extrinsic and instrinsic coupling strength is the key element for this criticality.

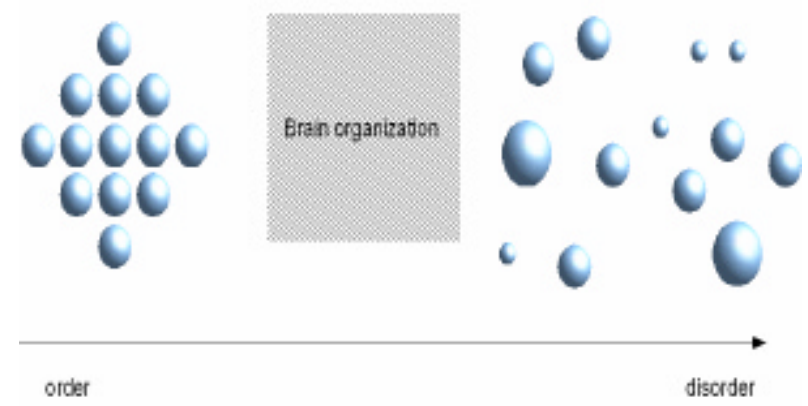

Fig. 2. criticality in self-organized systems. For high level of order (left), the system is excessively rigid and does not allow novelty and adaptation. For low level of order (right), the system is excessively unstable. Biological systems in general and brain systems in particular are postulated to live at intermediate stages of orders.

\section{RESILIENCE OF COGNITIVE STATES WITHIN THE EVER-CHANGING BRAIN}

The brain is a machine of very dynamic essence. At the same time than fluctuations and changes are required for adaptive and evolutive cognition, the plasticity of the brain is an issue to be addressed when one wants to implement there stable or reproducible cognitive states. Within its interconnected tissue, the configuration of neural activity is ever-changing: for two hypothetically perfect "iterations" of the exact same endogenous or incoming energy (eg. precise repetition of the same perceptual state, with the body in the same 
position, in a quiescent resting brain...), the configuration of neural activity would never be the same. Specific neurons may be available or refracted depending on recent activity. Neurons may have modified their susceptibility to the incoming energy due to plasticity at the synaptic scale. Hence, the coding in the brain can not be hidden in some cryptology of activity at the neural level.

Despite of those variations, the brain proves constantly its ability to realize functional equivalence, ie. the accomplishment of resilient cognitive state over changing configurations of neural activities. "In what is it surprising?", one may say, there is a colossal amount of neurons in the brain with spatially recursive mapping [16], when any one neural agent is not ready for its assignation, dozen may substitute themselves to ensure the job is done. The problem is that, stable patterns of neuronal activity can correspond to similar outcomes of cognitive states only in limited conditions.

The general rule in interconnected multi-element structures is that small initial differences may quickly explode in dramatically different outcome. What is needed to ensure the continuity of brain state in such situation? Two elements of response are proposed: First, attractors, or their remnants may allow the expression of stable cognitive state in a continuously changing neuronal substratum. In artificial neural networks, attractors have shown their resilience to partial or noisy informational source. Once established, the brain states are recovered even in noisy or occluded representation of the initial condition that preluded to its formation [17]. Second, the proper locus of the expression of cognitive states has to be postulated at the mesoscale rather than at the microscale [18]. There, the details of local activity do not matter any more, and emphasis is brought to the establishment of large-scale activity.

\section{LARGE SCALE COGNITIVE FUNCTION}

In line with a long tradition of single-cell neurophysiology [19], [20], [21], neuroscientists have been trying to understand the function of sensory brain areas by speculating on the tuning properties of their constituent neurons and their place on the hypothesized hierarchy of cortical areas. There, large-scale relations between brain areas do not matter as much as the activity expressed at the local scale, and how well the areas dynamically interact does not matter as much as how well they are anatomically wired together (hierarchical organization).

This theory does not explain well the strategy of the brain to slowly evolve or recombine its "coding preferences" from one area to the next. Taking the example of the visual system, we know many of the preferences of the neurons in each area of the occipital brain and we know that a giving feature to which a neuron responds vigorously in a given area will certainly be responded to some extend in the next area. We are not giving enough consideration to this slowly evolving mapping of stimulus preferences in successive brain areas. Why does the brain would spend some much energy and space to encode redundantly the same feature over and over again? What is fundamentally missed there is not what the neurons are doing in a given area, but how the brain uses the combinatorics of the states within successive areas to give sense to (sensory) information. Theories of large-scale brain function are able to implicitly capture this relational coding 
strategy, emphasizing the need for large-scale integration.

\section{INTEGRATION}

The large scale integrative tendencies of the brain have been captured by measuring transient increase in (linear) coherence between neurons or brain areas [22],[23],[24].

The precise nature of large-scale interaction, however, remains partially ununderstood. For long, the brain functioning was conceptualized as a serial transfer of information from an area to the next and so on. This theory does not resist to two major observations. First, the activity of brain areas is persistent [25], [26] and overlapping (eg. [27], [28], [29]. Second, long-range brain connectivity is typically [30], [31]. The feedback system is massive, 5 of the 6 cortical layers receiving top-down input by higher areas [32].

In consequence of this architecture, the activity of a brain area dynamically integrates reverberations of its activity with its persistent proper activity per se. It creates complex propagation patterns including maxima and minima, owing to the circulation loops with partner brain areas belonging to the same functional network. Those loops preclude the formation of brain rhythms, and their sequential recruitment and dissolution precludes the finer description of dynamical pattern formation and metastability.

Hence, a strong physical constraint in the expression of large-scale oscillatory activity between two electively connected local areas is provided by the pathway length, i.e. the size in millisecond of the loop linking related brain areas. It is suggested that longer-range interaction implements longer loops and relies on lower frequencies than short-range interaction [33]. Let us note however that each area is typically connected to more than a single other, therefore it is constrained by several characteristic periodicities. Those areas exert mutual influence that would be reconciled only for a finite number of their relative configurational states. Those possible configurational states are the brain patterns supporting cognition.

\section{SEgREGAtion}

As seen previously, integration has to be limited to prevent the loss of selectivity. The complementary requirement to large-scale integration is the preservation of the intrinsic activity expressed in the local areas (local segregation). Oscillatory behavior recorded at the (local) mesoscopic level is only circumstantially related to the intrinsic period of neuronal spiking events [34]-[37], [1]. Rather, the local oscillatory activity is an emergent property of varying neuronal activities and is typically idiosyncratic to each specific brain area. As seen both in pathological [13] and resting state, the attunement of local activity to the global oscillatory pattern prevents the expression of the specific activity supported by the parcellated brain areas and disrupt cognition. Therefore, mechanisms operating toward the preservation of local activity are to be present at the same time then mechanisms of binding between different areas. This fundamental requirement finds a ground in metastability, where those two complementary requirements were shown to be co-present.

VII. NESTED SYSTEMS IN SPACE AND TIME

A final postulate of the neuro-cognitive system 
is the lack of characteristic time-scale. The concept of function is used to refer to goal-oriented activity of the cognitive system. It is unspecified in its time-scale and degree of generality, and can encompass lasting and plural sets of cognitive operations such as those forming the maintenance in episodic memory of a multisensory experience and more elementary particles of cognition such as those recruited instantaneously during the retrieval of the name associated to a visually presented face. The brain functioning is flexibly adapted to these multiple spatio-temporal scales by introducing the concept of nested distributed modules [38] which are fitted neuroanatomically by postulating a granular or hierarchical organization [39]. Kelso [1] has shown that several cognitive behavior presented a power law scaling, ie. they can range a wide variety of time scale. Systems can be nested in time by their fractal scaling. There, the history of previous states, not only the current state, matters for the forthcoming activity with weak correlation between processes realized at different time-scale.

\section{REFERENCES}

[1] Kelso, J.A.S., 1995. Dynamic Patterns: The SelfOrganization of Brain and Behavior. MIT Press, 1995.

[2] Bressler, S.L., Kelso, J.A.S., 2001. Cortical coordination dynamics and cognition. Trends in Cognitive Sciences 5, 26-36.

[3] Friston, K.J. 1997. Transients, metastability, and neuronal dynamics. Neuroimage 5, 164-171.

[4] Fingelkurts, An.A., Fingelkurts, Al.A., 2004. Making complexity simpler: multivariability and metastability in the brain. International Journal of Neuroscience. 114(7), 843-862.

[5] Freeman, W.J., Holmes, M.D., 2005. Metastability, instability, and state transition in neocortex. Neural Networks 18(5-6): 497-504 (2005)

[6] Kelso, J.A.S., 1991. Behavioral and neural pattern generation: The concept of Neurobehavioral Dynamical System (NBDS). In H.P. Koepchen \&amp; T. Huopaniemi (Eds.), Cardiorespiratory and Motor Coordination, Springer-Verlag, Berlin.
[7] Kelso J.A.S., 1994. Elementary coordination dynamics. In: Swinnen SP, Heuer H, Massion J, Casaer P (eds). Interlimb Coordination: Neural, Dynamical, and Cognitive Constraints. Academic Press: New York. pp 301-318

[8] Murre, J.M.J., Sturdy, D.P.F., 1995. The connectivity of the brain: multi-level quantitative analysis. Biol Cybernet 73, 529-545.

[9] Sporns O., Kötter, R., 2004. Motifs in brain networks. PLoS Biology 2 (11), e369.

[10]Atlan, H., 1979. Entre le cristal et la fume. Paris, Seuil. [in french]

[11]Chialvo, D.R., 2004. Critical brain networks. Physica A 340 (4), 756-765.

[12] Szekely, G., 2001. An approach to the complexity of the brain. Brain Research Bulletin, Vol. 55, No. 1, pp. 1128.

[13]Kostopoulos, G.K., 2001. Involvement of the thalamocortical system in epileptic loss of consciousness. Epilepsia 42 (30), 13-19.

[14]Friston, K. J., 2000. The labile brain. III. Transients and spatio-temporal receptive fields. Philosophical Transactions of the Royal Society of London B 355, 253-265.

[15] Von Bertalanffy, 1968. General System Theory: Foundations, Development, Applications. New York: George Braziller.

[16] Mountcastle, V.B., 1997. The columnar organization of the neocortex. Brain 120, 701-722.

[17] Amit, D. J., 1995. The Hebbian paradigm reintegrated: Local reverberations as internal representations. Behavioral and Brain Sciences, 18, 617-657.

[18]Bressler S.L., Tognoli, E., in press. Operational principles in neurocognitive networks. International Journal of Psychophysiology.

[19]Granit, R., Svaetichin, G., 1939. Principles and technique of the electrophysiological analysis of colour reception with the aid of microelectrodes. Upsala Läkaref Färh. 65, 161-177.

[20] Galambos, R., Davis, H., 1943. The response of single auditory-nerve fibers to acoustic stimulation. J. Neurophysiol. 6, 39-58.

[21]Barlow, H. B., 1972. Single neurons and sensation: a neuron doctrine for perceptual psychology. Perception 1, 371-394.

[22]Gray, C.M., Singer, W., 1989. Stimulus-specific neuronal oscillations in orientation columns of cat visual cortex. Proc Natl Acad Sci USA 86: 1698-1702.

[23] Bressler, S.L., 1995. Large-scale cortical networks and cognition. Brain Research Reviews 20, 288-304.

[24]Rodriguez, E., George, N., Lachaux, J.-P., Martinerie, J., Renault, B., Varela. F.J., 1999. Perception's shadow: long-distance synchronization of human brain activity. Nature 397, 430-433.

[25] Colheart, M., 1980. Iconic memory and visible persistence. Perception and Psychophysics, 27: 183-228.

[26]Brody, C.D., Romo, R. Kepecs, A., 2003, Basic mechanisms for graded persistent activity: discrete 
attractors, continuous attractors, and dynamic representations, Curr. Opin. Neurobiol. 13:204-211.

[27] Nowak LG., Bullier J., 1997. The timing of information transfer in the visual system. In KS. Rockland, JH. Kaas, A. Peters, (Eds). Extrastriate visual cortex in primates, Vol. 12. New-York, Plenum Press.

[28] Schmolesky MT., Wang Y., Hanes DP., Thompson KG., Leutgeb S., Schall JD., Leventhal AG., 1998. Signal timing across the macaque visual system. Journal of Neurophysiology, 79: 3272-3278.

[29]Lamme, V.A.F., Roelfsema, P.R., 2000. The distinct modes of vision offered by feedforward and recurent processing. Trends in Neuroscience, 23 : 571-579.

[30]Felleman, D.J., Van Essen, D.C., 1991. Distributed hierarchical processing in primate visual cortex. Cerebral Cortex, $1: 1-47$.

[31]Lund, J.S., 2002. Specificity and non-specificity of synaptic connections within mammalian visual cortex. Journal of Neurocytology 31, 203-209 (2002)

[32]Robert, A. (1999); "Lamination and Within-Area Integration in the Neocortex", Doctoral Dissertation, University of California at San Diego, Cognitive Science Department.

[33]Basar, E., Basar-Eroglu, C., Karakas, S., Schurmann, M., 2001. Gamma, alpha, delta, and theta oscillations govern cognitive processes. Int. J. Psychophysiol. 39(2-3), 241248.

[34] Gray CM., König P., Engel AK., Singer W., (1989). Oscillatory responses in cat visual cortex exhibit intercolumnar synchroniation which reflects global stimulus properties. Nature, 338 (6213) : 334-337.

[35]Eckhorn R., Obermueller A., (1993). Single neurons are differently involved in stimulus-specific oscillations in cat visual cortex. Experimental Brain Research, 95 (1) : 177-182.

[36]Eckhorn R., Bauer R., Jordan W., Borsch M., Kruse W., Munk M., , Reitboeck HJ., 1988. Coherent oscillations : a mechanism of feature linking in the visual cortex. Multiple electrode correlation analyses in the cat. Biological Cybernetics, 60 (2) : 121-130.

[37]Varela, F.J., 1995. Resonant cell assemblies : a new approach to cognitive functions and neuronal synchrony. Biological Research, 28 (1), 81-95.

[38] Sutton, J.P., 1995. Neuroscience and Computing Algorithms. Information Sciences, 84:199-208.

[39]Changeux J.-P. (1996). Molecular mechanisms of synaptic transmission and its regulation: application to models of cognitive functions. NeuroImage 4: S7-S11. 